Archives

3| 1989

Varia

\title{
Le vitrail médiéval, un document pour l'historien
}

\section{Colette Deremble-Manhes}

\section{(C) OpenEdition}

\section{Journals}

Édition électronique

URL : http://journals.openedition.org/ccrh/2929

DOl : $10.4000 /$ ccrh.2929

ISSN : $1760-7906$

Éditeur

Centre de recherches historiques - EHESS

Édition imprimée

Date de publication : 15 avril 1989

ISSN : 0990-9141

Référence électronique

Colette Deremble-Manhes, "Le vitrail médiéval, un document pour l'historien », Les Cahiers du Centre de Recherches Historiques [En ligne], 3 | 1989, mis en ligne le 13 avril 2009, consulté le 19 avril 2019. URL : http://journals.openedition.org/ccrh/2929; DOI : 10.4000/ccrh.2929

Ce document a été généré automatiquement le 19 avril 2019

Article L.111-1 du Code de la propriété intellectuelle. 


\title{
Le vitrail médiéval, un document pour l'historien
}

\author{
Colette Deremble-Manhes
}

\section{Un nouvel art du récit en images : l'exemple de Chartres}

1 À la fin du XII ${ }^{\mathrm{e}}$ siècle, des incendies successifs ravagent une série de grandes cathédrales, - à Chartres le 10 juin 1194 -. En quelques années, il a fallu tout reconstruire. L'architecture se faisait alors révolutionnaire; de ce fait, la décoration intérieure - le vitrail en particulier - était entièrement renouvelée, dans son ampleur, dans son esprit esprit, ses thèmes, et son style.

2 Certes, on a pu réutiliser pour les nouvelles fenêtres des schémas anciens, des ensembles de verrières, ou seulement des panneaux, des bordures, voire des morceaux de verre réemployés en de tout autre contexte. On ne s'est pas complètement affranchi non plus des anciens programmes, dont on a certainement voulu reprendre l'essentiel. Il a fallu néanmoins créer, innover. Les dimensions et le nombre des ouvertures n'ayant pas de commune mesure avec ceux des anciennes constructions, les responsables du chantier se sont trouvés devant la nécessité d'imaginer des programmes nouveaux, donnant à la peinture sur verre, et à travers elle à l'art tout entier, une expansion étonnante.

3 L'audace des architectes permit l'épanouissement de l'iconographie. Parce que les grandes baies ne s'ouvraient pas seulement sur les façades, mais de façon systématique sur tout le pourtour de la cathédrale, l'imaginaire fut sollicité sans retenue. L'ampleur et l'importance des parois de verre proposées au peintre entraînèrent un renouvellement sans précédent des motifs et des formes, phénomène que ne connurent, en ce temps et dans cette mesure, ni la sculpture ni la peinture murale. Il y a dans cette soudaine explosion d'images une originalité qui appartient en propre à l'art du vitrail : en mettant à contribution l'inventivité des maitres du verre, l'évolution des techniques a conféré au vitrail un rôle moteur par rapport aux autres formes d'expression artistique. 
4 Les constructions sont rapides, l'oeuvre immense. Il faut aller vite, dans une fébrilité de création qui, tout à la fois, tient compte des enseignements passés, réutilise des modèles, se sert de techniques éprouvées, emploie des images héritées de constructions antérieures, sans pouvoir se dispenser d'innover, devant l'ampleur de la tâche. Par rapport aux oeuvres romanes du XII ${ }^{e}$ siècle, le vitrail, à partir des années 1200, n'a pas véritablement changé de nature. Pourtant sa généralisation oblige les concepteurs à prévoir des plans harmonisant les histoires selon un ordre et une cohérence autres, à repenser l'agencement des formes et des couleurs qui se succèdent désormais de façon continue dans l'espace de la cathédrale : la recherche de l'harmonie chromatique doit tenir compte d'un environnement où la couleur inonde les parois en d'incessantes contradictions, luttes d'influence, concordances. Parce que les ouvertures sont plus larges et plus nombreuses, les couleurs peuvent se faire plus denses, et le jeu narratif s'en trouve enrichi.

5 Pour que l'abondance ne suscite pas la monotonie et ne lasse pas le regard, on redouble d'inventivité pour varier les schémas formels, et la structure des récits s'en trouve remodelée. On doit refondre le flux narratif en masses distinctes, trouver de nouvelles cohérences de l'ensemble du programme iconographique, comme de chaque récit dans sa particularité. L'élaboration de schémas géométriques infiniment variés casse la rigidité des panneaux anciens, de ces formes orthogonales où, comme dans le vitrail chartrain de la Passion du XII ${ }^{e}$ siècle, les cercles ne s'inscrivaient que de façon factice dans leur cadre quadrangulaire, ne laissant aucune place à l'intérieur de la fenêtre à la décoration. Celleci échappe aux bordures, qui se font plus réduites, et se loge dans les interstices nombreux et variés laissés libres par l'entrecroisement des motifs : un nouveau langage s'instaure dans ce rapport incessant qu'entretiennent formes et figures au sein même de la fenêtre. L'histoire se déchiffre différemment, et la nouveauté de l'organisation formelle en permet une lecture plus raffinée.

6 Au sein de cette révolution, la cathédrale de Chartres apparaît à bien des égards comme un phare, par la densité de ses images et l'originalité de leur organisation. L'exceptionnelle conservation de l'ensemble médiéval y rend possible plus qu'ailleurs une reconnaissance de ce que fut l'ambition iconographique de ces vaisseaux d'images.

7 Les chercheurs, dans le domaine du vitrail, ont été le plus souvent absorbés par la nécessité de dater, de répertorier des oeuvres très détériorées par le temps: travail primordial qui conditionne tous les autres. Les études de style ont parallèlement été menées avec une grande érudition. Sans doute l'iconographie est-elle encore restée quelque peu le parent pauvre de ces recherches, dans la mesure où elle est entièrement dépendante des conclusions de l'analyse archéologique. Parce que les travaux de critique d'authenticité menés actuellement à Chartres conduisent à la conclusion globale de l'excellente qualité de la conservation de la vitrerie chartraine (dans la nef en tout cas), une recherche de type iconographique parait pouvoir être menée sans risque. Elle permet l'utilisation, à ce jour très peu expérimentée, des images du vitrail comme documents pour l'historien, sources d'une exceptionnelle éloquence.

8 Le terrain de cette étude se limite aux verrières basses de la cathédrale chartraine, et aux quelques onze cents scènes qu'elles déploient : ces verrières en effet constituent un tout homogène quant à leur structure, leur contenu narratif, leur dimension, leur date. Les fenêtres hautes, dans leur ensemble plus figuratives que narratives, méritent d'être étudiées séparément. 


\section{Le temps de la construction chartraine}

les images dans les circonstances qui leur ont donné naissance : temps, événements ou personnes; celle, sans doute essentielle, de Pierre de Celle, dernier grand évêque intellectuel de Chartres avant cette autre figure, politiquement déterminante, que fut l'évêque bâtisseur, Renaud de Mouçon ; celle enfin, si controversée, de Pierre de Roissy, chancelier du Chapitre dès les premières années du XIII ${ }^{\mathrm{e}}$ siècle.

En ce qui concerne les dates, la tâche reste difficile dans la mesure où il n'est guère possible de dater la décoration intérieure indépendamment de sa structure de pierre, et où la recherche d'une chronologie absolue paraitt quelque peu de l'ordre de l'utopie. Sur ce point la position des historiens reste conflictuelle, et il ne s'agit pas ici d'apporter des hypothèses définitives. L'étude iconographique, en particulier celle de la représentation des sacrements, parle souvent en faveur d'une date antérieure au concile de Latran IV. Par ailleurs le poème de Guillaume le Breton, sur lequel s'appuient beaucoup de critiques pour fixer la fin de la construction générale de la cathédrale à 1220, est, selon toute vraisemblance, achevé vers 1214, comme le montre Delaborde, qui édite en 1882 les oeuvres de l'historiographe. Quant aux verrières du choeur, si nombreuses, attribuées au maitre de Saint-Chéron, elles semblent passablement post-datées sur la foi d'une réflexion hâtive de Merlet au siècle dernier. Ces deux petits sondages, qui ni l'un ni l'autre bien sûr ne conduisent à des conclusions certaines, ont seulement pour prétention de réduire l'assurance d'une fourchette de dates s'étendant de l'incendie de 1194 à 1230, et ouvrent l'hypothèse d'une élaboration plus rapide, plus groupée, de l'ensemble des verrières basses.

11 Plus importantes que les chiffres, sans cesse remis en cause, les concordances que l'on peut établir entre des images et les étapes de la vie de l'esprit. La spéculation théologique et philosophique connaît une certaine pause en cette fin $\mathrm{du}$ XII $^{\mathrm{e}}$ et ce début du XIII ${ }^{\mathrm{e}}$ siècle, entre la première et la seconde scolastique. La mort de Jean de Salisbury, en 1180, clôt une période d'intense activité de l'école chartraine. Les écoles de Laon et de Reims ont aussi perdu leur éclat ; l'heure des moines et des chanoines s'éteint pour faire place à des productions séculières, parisiennes: mutations des personnes, des objets, des lieux, temps de germination et de restructuration de la pensée, qui occupe Paris jusque vers 1230. C'est dans cet entre-deux qu'à Chartres, Bourges, Sens... va germer une nouvelle forme de spéculation et d'expression théologique à travers le langage iconique, qui prend pendant un demi-siècle le relais de pensées plus abstraites. C'est un peu comme si les programmes monumentaux des cathédrales avaient pris le pas sur le langage articulé et monopolisé pendant quelques décennies les chemins de la réflexion.

Ce moment voit l'affirmation d'un grand renouveau pastoral. L'Église d'Occident traverse une période de mission et de réorganisation, au cours de laquelle le message chrétien doit être adapté au nouveau visage des villes et aux nouveaux besoins des laïcs. On prêche de plus en plus en langue vulgaire, on oriente le discours pour lutter contre les déviances qu'entraîne inévitablement la progression de la langue romane, on adapte le répertoire hagiographique à cette nouvelle pastorale. Le travail du vitrail se situe dans ce sillage de rénovation, il en est même un des rouages essentiels. Il s'affirme au lieu où se croisent le souci d'ouverture de clercs de plus en plus intégrés dans le monde et le désir d'expériences spirituelles d'une élite laïque et cultivée. Il prolonge l'effort verbal de 
vulgarisation en un moment où l'on pense avoir fait le point sur la doctrine chrétienne et avoir durablement réglé la pratique religieuse. Le souci extrême porté dans les vitraux à la vie sacramentelle, qui est précisément le point le plus travaillé lors des assemblées synodales de ce temps, est l'une des marques du lien qu'il faut voir entre la vie conciliaire et les programmes iconographiques des cathédrales. L'art du vitrail, par l'ampleur et la quantité des images proposées, permet d'exposer en détail, mieux que les sculptures, déterminées par davantage de contraintes, et plus marquées par la tradition, les décisions que l'on veut faire connaitre. Il se présente alors comme une voie de transmission, privilégiée et permanente, de la doctrine.

La période de la construction de la cathédrale coïncide avec le « règne » d'Innocent III. L'impulsion très forte qu'il donne à la vie ecclésiale par sa personnalité hors du commun est déterminante pour le projet chartrain. Conçue quelques années avant cet événement si essentiel pour l'histoire du monde chrétien que fut le quatrième concile de Latran, l'iconographie chartraine en annonce les grandes décisions. C'est dans la tension entre les cadres juridiques établis par Gratien, l'exaltation mariale héritée de saint Bernard, l'ouverture culturelle qui est la marque de l'école de Chartres, les préoccupations pastorales commandées par la lutte contre les hérésies, l'esprit réformateur imprimé par la personnalité d'Innocent III, que s'épanouit l'ingéniosité exceptionnelle des créateurs chartrains. Plus qu'à Canterbury, très marquée par son appartenance monastique, plus qu'à Bourges et à Sens, moins directement centrales dans cet entrecroisement d'influences, Chartres se trouve au point de convergence d'un passé extraordinairement riche et d'enjeux modernes qui transforment le visage de l'Église.

Ces enjeux sont aussi ceux du tournant que connaît à l'aube du xiII siècle la littérature hagiographique. Or l'iconographie chartraine est, plus que d'autres, déterminée par l'hagiographie. Dans les bréviaires, les leçons se réduisent faute de place et se limitent parfois à quelques lignes résumées. L'hagiographie échappe au domaine de la liturgie pour s'épanouir en d'autres formes moins contraignantes, plus accessibles. C'est le moment où l'on entreprend la transformation du corpus hagiographique par un vaste mouvement de traduction en prose française. Mouvement dont on connaît l'ampleur, mais dont on n'a pas encore à ce jour analysé la portée littéraire, pourtant considérable. Ce mouvement suit l'élan de construction des grands ensembles de vitraux, puisque les premières traductions isolées apparaissent vers 1210 , et il ne se généralise qu'au milieu du XIII ${ }^{e}$ siècle où il prend un essor considérable. Les rédactions en prose des légendes vont de pair avec une intellectualisation du message, le récit s'adressant davantage à des laïcs cultivés et à des lecteurs qu'à des auditeurs. On a souvent parlé de l'importance du passage d'une littérature avant tout destinée à être entendue à une autre proposée à la lecture, et de l'écart qualitatif qui distinguait ces deux types de production littéraire. Dans l'entre-deux il semble que s'intercale un troisième type de production, celle qui n'est destinée ni à être entendue, ni à être lue, mais à être vue. Peut-être est-ce ce jalon de la visualisation qui a permis l'appropriation des textes par des individus capables de lire parce qu'ils avaient vu. L'épanouissement des vies de saints dans le vitrail, qui est l'expression d'un si important mouvement de traduction, d'analyse et d'intellectualisation de la littérature hagiographique, a contribué à faire naître le désir de réentendre ces vies dans la langue vulgaire. 


\section{La notion de programme iconographique}

15 Une des grandes questions concernant le vitrail chartrain porte sur sa cohérence. La notion de programme occupe dans l'esprit de ce temps une place essentielle: parce que l'univers est conçu comme un tout, et que la cathédrale figure un microcosme rival ou miroir de la création divine, les artistes ont l'ambition de reproduire cette universitas dans leurs compositions architecturales et iconographiques. Peut-on dans un tel contexte se résoudre à croire, comme on l'a dit jusqu'ici, que les théologiens chartrains ont laissé bâtir leur cathédrale selon les lois du hasard ou des seules pressions locales ? Comment justifier un tel décalage entre le programme sculpté, que tous s'accordent à reconnaître érudit et pédagogique, et le programme vitré qui lui est contemporain? Il n'est guère de lieu où l'esprit de synthèse se soit exercé avec autant de force que dans l'Ecole qui a fait au XII siècle la gloire de Chartres. Il n'est guère de lieu qui ait autant attiré les regards des foules. Il n'est guère de construction au Moyen Age qui ait suscité tant d'attention, de ferveur, tant drainé de fonds, tant nécessité d'ingéniosité et d'intelligence : a-t-on pu négliger ce qui fait la fierté des théologiens et des artistes, l'ordonnance des données de la foi en images hiérarchisées, combinées, progressives, typologiques? Ne convient-il pas, au contraire, malgré la rareté des informations précises, malgré les lacunes de l'iconographie, de faire crédit à l'esprit synthétique de ces héritiers de la première scolastique, et de tenter de redécouvrir leur message, fût-il aujourd'hui lacunaire?

La quête de cette cohérence hypothétique peut se faire dans sa coïncidence avec d'autres modes d'expression : homélies ou écrits théoriques parlent, de façon très semblable aux verrières, de Noé, de Joseph ou des Apôtres, et dans la force de ces discours quelque chose du discours iconique, peut-être, est révélé. L'ensemble des textes littéraires et théologiques qui créent le tissu culturel où les vitraux sont nés, dessinent le premier système englobant où se situent les verrières basses de Chartres. Il importe donc de mettre en relation des écrits et des images, de trouver les effets de résonance de ces dernières au sein d'une pensée symbolique.

Il est évident que ces correspondances ne relèvent pas de relations de cause à effet ou d'automatismes logiques. Il est moins question d'énoncer les clefs d'un éventuel programme dans son adéquation parfaite avec tel ou tel discours que de désigner un certain nombre d'affinités signifiantes entre deux types d'expression voisins. C'est dire qu'il faut chercher, pour chaque thème utilisé dans les verrières, dans une lecture la plus large possible de la patrologie, la manière dont il était reçu au Moyen Age. Cette enquête sur la réception des motifs hagiographiques apporte un éclairage précieux sur les liens qui unissent dans la pensée médiévale les motifs rapprochés dans l'espace de la cathédrale. Dans cette organisation spatiale, la trouvaille des chartrains paraît se situer dans l'importance donnée au motif apostolique dans l'axe de la cathédrale: ce changement de perspective, qui révèle la nouvelle détermination de l'ecclésiologie proche de la pensée d'Innocent III, a dû paraître révolutionnaire au temps de la construction. Pour le reste les rapports de contiguïté, les regroupements partiels, font, de proche en proche, parler le livre-cathédrale à la manière d'un recueil de sermons. 


\section{Chartres et les autres programmes vitres du Moyen Age}

18 Le principe même d'harmonie implique que la recherche des clefs de l'intelligibilité d'un programme particulier se fasse à l'intérieur d'autres ensembles, d'autres cohérences. L'harmonie dessinée par toutes les images de la cathédrale chartraine, sculptures et vitraux, et celle des autres programmes vitrés contemporains constituent les ensembles signifiants à l'intérieur desquels les verrières basses trouvent un sens. L'usage de l'informatique permet de comptabiliser, de classer, de hiérarchiser des images trop nombreuses pour être saisies en dehors de cet outil. Ce sont plus de 2000 scènes sculptées ou peintes de la cathédrale, et quelque 800 thèmes des verrières légendaires médiévales qui peuvent aider à placer les images des verrières basses en situation relative, à chercher, par une énumération sérielle, des taux de fréquence, des dominantes, à repérer quasi visuellement la particularité chartraine.

L'inventaire de l'iconographie de la cathédrale dans son ensemble énumère le projet décoratif proposé au terme du chantier qui, depuis 1194 jusqu'au milieu du xIII ${ }^{\mathrm{e}}$ siècle, en un ensemble intégrant les traces romanes épargnées par l'incendie, échafaude un corpus très peu modifié par la suite. Il fait apparaître, à travers un certain nombre d'échos, la grande indépendance de l'expression peinte par rapport à l'expression sculptée, ou plutôt les fonctions bien différentes des façades par rapport à l'intérieur.

Le répertoire thématique des verrières légendaires médiévales se heurte à la difficile distinction des scènes et des figures, à la dispersion du corpus, à l'imprécision des études qui en sont faites et à la difficulté de se conformer au système de conventions du Corpus Vitrearum, peu adapté à une analyse iconographique. Par delà ces problèmes, les conclusions de l'analyse mettent en exergue les orientations très nettement politiques de Chartres, et sa détermination moderniste. L'ensemble des vitraux chartrains se trouve ainsi campé dans l'horizon de créations voisines, rivales, ou plus lointaines, qui lui donnent une sorte de relief. Certes, l'état terriblement lacunaire dans lequel nous parvient l'art du vitrail ne permet pas d'établir d'indices de fréquence parfaitement fiables des récits dans ce corpus trop mutilé et trop incertain dans ses reconstitutions. Néanmoins le tableau synthétique que l'on peut en dresser permet d'évaluer un ordre de grandeur évocateur de la réalité perdue. Il est, en tout état de cause, d'une grande utilité pour imaginer avec une certaine vraisemblance le paysage iconographique dessiné par la décoration de la peinture sur verre. Il permet surtout de situer le dessein chartrain à l'intérieur d'une trame diachronique, et de saisir cette création dans un contexte évolutif.

21 Pour l'Ancien Testament, on constate dans l'ensemble un spectre peu étendu, réduit à vingt-deux thèmes, dont cinq essentiels ; un usage majoritaire avant 1200 de la typologie ; et un intérêt tardif pour la narrativité vétérotestamentaire. Pour le Nouveau Testament, la prédominance des Passions, et, à l'intérieur de ce cycle de l'art typologique des Vies du Christ, par opposition à la faiblesse des Jugements derniers et des Apocalypses, délimite un paysage iconographique bien propre au vitrail par opposition à la sculpture. Pour l'hagiographie enfin, le triomphe de saint Nicolas, qui apparaît comme le héros de cette iconographie médiévale, l'ouverture faite aux traditions locales, et les tentatives timides d'intégration de la modernité sont les traits dominants de la thématique du vitrail légendaire. 


\section{L'héritage hagiographique} comparatiste, l'analyse plus précise de la thématique chartraine. Les verrières ne sortent pas ex nihilo du pinceau de maîtres de génie. Elles trouvent leur fondement dans une longue tradition hagiographique et iconographique.

23 À propos des sources écrites, les lectionnaires liturgiques sont loin d'être la clef de la création du vitrail, comme on le croyait après l'œuvre magistrale de Delaporte. Les vitraux chartrains ne sont pas le décalque servile de lectures quotidiennes: les choix iconographiques au contraire relèvent d'une culture vaste, ambitieuse, calculatrice, ingénieuse, inventive autant que conformiste. Quelques sondages, à partir entre autres des histoires de saint Lubin, de saint Eustache ou de saint Julien, suffisent à montrer l'ampleur du génie créateur chartrain, celui qui réveille des histoires endormies, rejoint le vent de la mode, suscite des formes nouvelles. Une des grandes gloires des imagiers de la cathédrale est d'avoir su réunir, sur ces parois de lumière, des récits issus d'horizons très divers, que, pour un grand nombre, les lectionnaires locaux ignoraient, ou évoquaient de manière insuffisante pour satisfaire leur sens pictural et littéraire. Cet assemblage, qui a nécessité de leur part une enquête hagiographique approfondie, une curiosité et une ouverture d'esprit exceptionnelles, en même temps qu'une perception sûre des grands courants de l'actualité, relève de la création littéraire plus que de la compilation de chanoines confinés dans les lectures quotidiennes des leçons liturgiques.

Les vitraux légendaires de Chartres, qui dépassent sans doute en ampleur, en nombre et en dimension tous ceux qui les ont précédés, constituent le premier recueil de Legendae novae où l'on rassemble, pour les publier, les traditions orales ou écrites stratifiées depuis parfois des siècles. L'exposé de leurs contenus thématiques permet de comparer ces deux types de vulgarisation, pour lesquelles le vitrail se montre précurseur.

anture intellectuelle de haute volée de cette écriture a su redonner du lustre à certains cultes liturgiques, assurer une publicité durable à des saints dont on possédait les reliques, intégrer dans le panthéon chrétien des figures quelque peu marginales : bref c'est une mise à jour d'un héritage hagiographique. Le vitrail chartrain publie un véritable tableau de la mode hagiographique au début du XIII ${ }^{e}$ siècle.

L'exposition des figures de sainteté est loin d'être linéaire. Une des manières les plus évidentes d'établir des hiérarchies reste le positionnement des unes et des autres à l'intérieur d'un espace très codifié ; il en existe d'autres, plus discrètes. Le nimbe en est un. Il est bien difficile, dans une étude systématique de la présence ou de l'absence du nimbe, de conclure à une logique dont on aurait les clefs. Il faut aussi compter avec les incertitudes des restaurations, et avec les habitudes iconographiques qui peuvent diverger entre des verrières voisines, voire à l'intérieur d'une même verrière. Des incohérences demeurent inexpliquées. Pourtant il n'est pas neutre de savoir qu'Eustache, ce saint laïc, n'est jamais gratifié d'une auréole, que Marie-Madeleine n'en porte pas lorsqu'elle personnifie la pécheresse prosternée aux pieds du Seigneur, qu'Antoine attend d'être effectivement ermite, et Lubin d'être accueilli dans son monastère, pour être nimbés, alors que Martin avant même sa conversion et son baptême, a la marque de la sainteté, que Nicolas ou Sylvestre sont nimbés dès leur plus tendre enfance, contrairement à Julien, qui ne reçoit l'auréole que lors de sa vie de service. Un des cas où

Les Cahiers du Centre de Recherches Historiques, 3 | 1989 
l'hésitation sur la sainteté est la plus évidente pourrait être Charlemagne, ce saint si récent dont la canonisation irrégulière ne fut pas acceptée sans difficulté par l'Église, présenté alternativement avec et sans son auréole.

La sainteté donc n'apparaît pas ici comme un diplôme unique et homogène : elle a un visage différencié, ordonné, conjugue la tradition et l'audace.

\section{L'originalité des images chartraines}

Bien souvent ce sont les images qui engendrent les images : il importe de vérifier dans quelle mesure les maîtres chartrains ont trouvé des éléments d'inspiration dans des oeuvres antérieures, et surtout, car cette dernière tâche a un caractère à la fois infini et invérifiable, quelle fut leur véritable originalité dans le choix des motifs, leur agencement, leur développement. Les interférences entre les images du vitrail et celles des miniatures sont peu importantes. L'art du vitrail semble avoir créé son propre domaine, et inventé son autonomie iconographique dans l'expansion sans précédent des motifs hagiographiques.

$\mathrm{Au}$ contraire, les vitraux présentent entre eux de nombreuses similitudes iconographiques. La proximité des programmes, la ressemblance de certaines images confirment l'idée d'une importante circulation de projets entre les différents lieux de création iconographique. Bien des cycles hagiographiques chartrains ont sans doute trouvé leur première représentation importante dans l'art du vitrail : sainte Catherine à Angers, saint Vincent à Saint-Denis, à Angers et à Charentilly, saint Jean l'Evangéliste et saint Eustache à Sens, saint Etienne à Châlons-sur-Marne et au Mans.

Beaucoup de ces cycles ne nous sont conservés qu'à Chartres, ce qui valorise grandement l'importance de la cathédrale dans l'histoire de l'iconographie hagiographique: saint Théodore, saint Pantaléon, saint Antoine, saint Paul ermite, saint Appolinaire, saint Julien l'Hospitalier, et bien sûr les saints locaux comme saint Lubin ou saint Chéron. D'autres naissent simultanément à Chartres, Sens et Bourges, comme l'illustration de la vie de saint Thomas ou de saint Jacques (du moins pour Bourges), à Chartres et à Angers, comme celle de saint André. Partout Chartres l'emporte en nombre de scènes illustrées et en qualité de conservation. Toujours le sens créateur des chanoines est mis à l'épreuve : bien des tableaux ne se trouvent qu'à Chartres, même dans les motifs hagiographiques les plus classiques. La cathédrale s'impose ainsi comme un lieu d'innovation artistique et spirituelle extraordinaire.

\section{Une certaine réticence face à la lecture typologique}

31 Par l'ampleur des informations qu'ils fournissent sur la vie médiévale, les vitraux chartrains peuvent être étudiés dans leur fonction de miroir, non pas seulement au sens trop passif de plaque réfléchissante, mais au sens médiéval de modèle. Les créateurs chartrains se servent du vitrail comme d'un outil d'écriture et de pensée qui leur permet d'innover, non pas seulement de traduire une mentalité, mais de la révéler, de lui donner une forme inédite. C'est en ce sens qu'il paraît nécessaire de renverser les rapports de création rivale qu'entretiennent l'image et le texte. Ces effets de miroir méritent surtout d'être observés dans le domaine de la spiritualité et dans celui de la politique, deux pôles qui ont fortement sollicité l'intelligence des chanoines créateurs. 

typologique qu'il ne s'intègre à l'intérieur de la démonstration globale faite à travers l'ensemble du programme de la cathédrale : la verrière décrit un itinéraire qui conduit du péché à son effacement, de la débauche à l'alliance, grâce à la médiation de l'arche centrale, bateau-église stable qui traverse les flots de la mort. La construction très ferme et très maîtrisée de la verrière épouse avec précision la trame du texte biblique, tout en modifiant la logique finale. Le génie chartrain est d'avoir su traduire, avec un sens narratif sûr, la saveur dramatique de la Genèse, de l'avoir exprimée en des termes picturaux éloquents, avec cette progression de la crue et de la décrue, avec cette invasion du désastre, avec cette répétition des images qui évoque la lenteur lancinante des jours et des mois de déluge, tout en l'encadrant à l'intérieur de la dynamique du péché et de l'alliance. La fidélité au texte va jusqu'à l'audace de la représentation de l'épisode étrange des Néphilim; la perfection plastique va jusqu'à l'élégance sordide de ce rapace courbé sur la tête d'un noyé dont il pique les yeux clos; l'exigence théologique va jusqu'à l'élimination de la faute de Noé et à l'inversion de la suite logique du texte biblique : Noé doit rester juste pour sauver l'humanité, et l'arc, prolongement et renversement graphique de toutes les figures d'arches au centre de la verrière, est dressé entre Dieu et son peuple. Enfin le désir de prédication exemplaire va jusqu'à reconstruire le récit du déluge autour du thème du couple, couple pervers et violent d'avant la rencontre de Dieu et de Noé, couples d'hommes et d'animaux qui entrent par paires dans le baptême de la mort, et qui en sortent pour être bénis et promis à la fécondité.

Les commentaires patristiques à la manière de ceux d'Isidore de Séville sont gommés dans le vitrail, tout entier consacré à une narration linéaire et exemplaire, où les développements plastiques l'emportent sur la construction symbolique. Cela n'empêche pas la pensée théologique de s'affirmer avec force, celle d'une Église qui se construit, 
suffisamment stable pour traverser la tourmente, qui sait bénir et maudire pour faire alliance avec Dieu. La puissance de l'iconographie chartraine réside dans sa capacité à construire des récits unifiant des épisodes que l'on rencontre isolés dans la tradition iconographique. Autre permanence, celle qui aménage, dans le centre de la verrière, des panneaux, rehaussés par leur forme - des carrés posés sur pointe - et par leur place, qui disent l'essentiel du message : ordre de Dieu, construction de l'arche, déluge, envoi de la colombe, sortie de l'arche, malédiction de Cham, alliance avec Dieu. Les panneaux secondaires par rapport à ce centre développent les circonstances et accumulent les détails.

37 C'est davantage sur cette recherche narrative et théologique que s'est centré le talent des artistes.

\section{La dévotion mariale}

La foi est aimantée par des pôles en proie à une lente dérive. Celui que je prendrai ici pour témoin est le pôle marial. Il est particulièrement signifiant à Chartres, consacrée à NotreDame. Mais il ne l'est pas moins dans le contexte plus large de la recherche spirituelle depuis saint Bernard et Abélard, depuis l'éclosion d'une dévotion populaire envers la figure féminine qui commande l'Incarnation, depuis l'affinement d'une pensée qui cherche à intégrer la Vierge dans une vaste construction dogmatique.

A Chartres, deux oeuvres magistrales, séparées d'une cinquantaine d'années, illustrent, dans la sculpture et dans le vitrail, la vie de la Vierge. Bien différentes, dans leur conception iconographique et idéologique, elles contribuent de manière décisive à asseoir la tradition iconographique de l'Evangile de la Nativité en Occident. La force de la sculpture a été d'imposer de manière éclatante une légende encore suspectée. Lorsque les maîtres verriers réécrivent l'histoire au début du XIII ${ }^{e}$ siècle, il s'agit davantage de conférer un statut officiel, liturgique, à la légende. Ils omettent pour cela les épisodes ou les détails à la saveur toute familière (pleurs de Joachim et d'Anne après le refus des offrandes, pleurs de Joachim dans le désert, geste de tendresse après leurs retrouvailles, bain dans un baquet, présentation au temple, retour à Nazareth, attente commune de l'Annonciation), et insistent sur l'orthodoxie rituelle et théologique de la légende. Enfin on intègre le récit à la fois au modèle de la vie du Christ qu'il précède et aux modèles hagiographiques qu'il côtoie dans la cathédrale, et auxquels il devient très homogène : la légende est à la fois ainsi banalisée et formalisée. Il s'agit moins d'un honneur, un peu provoquant comme a dû l'être l'imposante frise des chapiteaux, rendu à la Vierge, que d'une entreprise de liturgisation d'un récit apparaissant aux théologiens intellectuels du début du XIII siècle comme inutilement familier. Tel est le travail capital d'adaptation pour lequel les théologiens chartrains semblent avoir eu peu de modèles intermédiaires.

Le choix de la légende de la Dormition de la Vierge pour une verrière de la cathédrale n'est ni exceptionnel ni novateur, mais il correspond à un intense moment de mutation doctrinal à l'intérieur duquel les verriers chartrains ont su imprimer leur marque originale. Le soin et la précision avec lesquels est traité le récit chartrain sont étonnants et témoignent du crédit très grand fait à la continuité narrative : tous les détails du texte sont scrupuleusement observés, et la légende reçoit dans le vitrail sa première illustration de grande envergure. En même temps que le récit est articulé avec netteté en ses différentes phases, grâce à l'isolement de chaque motif en un tableau particulier, la 
pensée théologique trouve une clarté superbe en cette démonstration parfaitement menée, depuis la distinction des Assomptions corporelles et spirituelles jusqu'à l'affirmation du co-regnum du Christ et de la Vierge, depuis l'habile répartition des rôles de Jean, Pierre et Paul, qui a tant divisé la chrétienté, jusqu'au rattachement de la légende au mythe dionysien, depuis le symbolisme de l'Arche d'Alliance jusqu'à la tension entre la vénération et le refus de croire du grand-prêtre, c'est-à-dire à l'opposition des juifs et des Gentils. Enfin, par-delà la perfection d'une écriture narrative et théologique si dense, de la vibration des anges qui adorent et encensent, des couleurs très homogènes à l'intérieur d'une palette volontairement peu étendue, des compositions toujours symétriques, une harmonie liturgique s'empare de ces images pour en faire un chef-d'oeuvre rare.

\section{Vitrail et merveilleux}

41 La foi délimite le champ du christianisme dans sa permanente tension avec le champ du religieux, du mythique. Les théologiens doivent sans cesse se garder de contaminations possibles avec le merveilleux païen. Un des exemples du savant équilibre qu'ont su ménager les maîtres chartrains peut se lire à travers le vitrail de saint Jean l'Evangéliste. La version qu'ils donnent de la légende ménage ses aspects anecdotiques, voire ambigus dans son lien avec l'alchimie, en même temps qu'elle la restructure très fortement par des effets esthétiques, et surtout par une détermination théologique et liturgique forte. Le vitrail de Chartres, qui est sans doute le plus ancien témoignage iconographique que nous ayons conservé en Occident d'un cycle extensif sur la vie de saint Jean, procède d'une décision audacieuse : présenter, sur les murs d'une cathédrale, une vie entièrement apocryphe, dont les Pères s'étaient beaucoup méfiés, et qui n'avait, peut-être pour cette raison, jamais suscité de représentation importante. Audace théologique: il s'agit d'infléchir l'histoire dans un sens liturgique, spirituel, voire mystique, tout en lui conservant son emprise sur l'imaginaire populaire. Audace iconographique dans la mesure où la composition d'ensemble, l'organisation et le choix des scènes, la structure des images sont inédits et témoignent d'une véritable création artistique. Le traitement de l'histoire de Jean l'Evangéliste reflète les variations d'attitude de l'Église face à l'étrangeté de certains récits déviants. La légende présente trois épisodes problématiques, en ce sens qu'ils appartiennent à la tradition gnostique dont la légende est issue à l'origine: reconstitution des pierres précieuses brisées par les disciples de Craton, transformation du bois en pierres précieuses, épisode de la coupe empoisonnée. Les deux premiers appartiennent à la tradition alchimiste, le troisième évoque davantage des scènes de sorcellerie. A l'intérieur d'une histoire très fertile en événements divers, relevant de la mission, de l'apostolat, de la confession, du témoignage par le martyre, et de l'imitation du Christ, le choix par les artistes de ces épisodes, au détriment d'autres plus spécifiques du modèle chrétien, est significatif. A peu près au même moment on a pu faire des choix aussi opposés que ceux de Lyon, où toute allusion à l'alchimie est évacuée.

Les figures monstrueuses, si abondantes dans la sculpture romane, s'inscrivent aussi à la même époque dans la peinture sur verre, bien que plus discrètement : à part la verrière dionysienne des griffons, on trouve surtout de discrètes figures de monstres à l'intérieur des bordures. Les verrières romanes d'Angers en étaient particulièrement riches, avec ces quadrupèdes ailés à tête humaine ou à tête de griffon de la verrière de l'Enfance du Christ, ou ces êtres hybrides enroulés dans les feuilles de palmette de la vie de sainte Catherine. Alors que les verrières chartraines $\mathrm{du} \mathrm{XII}^{\mathrm{e}}$ siècle cernent aussi les récits de 
bordures où la végétation stylisée rivalise avec les inventions décoratives les plus raffinées des initiales ornées des manuscrits, et enserre dans ses entrelacs savants animaux monstrueux, oiseaux mythiques ou quadrupèdes imaginaires, celles $d u$ XIII $^{\mathrm{e}}$ siècle, beaucoup plus sobres, adoptent de sages motifs géométriques. Signe de la jugulation du merveilleux?

Les légendes, tenaces au $\mathrm{VI}^{\mathrm{e}}$ siècle, de saints ayant eu à combattre des dragons, symbole du paganisme détruit par le christianisme, ne trouvent pas d'écho dans les vitraux du XIII siècle. Ni saint Georges, ni saint Marcel, saint Lô, saint Loup ou saint Germain d'Auxerre, dont les légendes sont très populaires au Moyen Age, ne sont représentés dans cette tâche. Les compilateurs des environs du $\mathrm{VII}^{\mathrm{e}}$ ou VIII ${ }^{\mathrm{e}}$ siècle de la légende de saint Théodore enjolivent son histoire en y ajoutant un motif de combat contre un dragon. Sur ces broderies fantastiques, le vitrail reste silencieux. Est-ce par manque de place, comme le suggère le chanoine Delaporte à propos de la légende de Sylvestre ? La raison est faible, surtout quand on doit la répéter si souvent au sujet précisément de ces mêmes motifs fantastiques, et surtout aussi quand on lit, dans la même verrière, bien des scènes inutiles pour la stricte logique narrative. N'est-ce pas plutôt le signe d'une détermination de la part des concepteurs? Détermination qui consiste à épurer les légendes d'un côté mythique qui embarrasse une église fort intellectuelle, et désireuse de s'affranchir du poids de paganisme véhiculé par ces légendes.

Tout en préservant sa part au fantastique, les créateurs du vitrail se refusent à s'écarter des réflexions théologiques où la pensée a mûri, faisant jour à une conception personnelle et intérieure du péché. Le démon reste une manière commode de visualiser le mal, et d'exprimer la profondeur d'une réflexion sur le péché et la pénitence. De manière générale, la méfiance des intellectuels chartrains s'exerce par rapport aux excès des figurations merveilleuses ou monstrueuses, et le surnaturel s'exprime davantage en des formes théologiquement très élaborées et rares, comme la hiérarchie des anges.

\section{Vitrail et politique}

Enfin le plus important peut-être de ces miroirs innove une théorie du politique. Il n'est guère de temps où le politique et le religieux soient aussi intimement mêlés : les verrières parlent des rois, parlent aux rois. Elles écrivent un vaste speculum principis où les problèmes de la succession du pouvoir, bien d'actualité au temps de Philippe Auguste, celui des rapports du prophétisme et du gouvernement, celui de l'héritage carolingien ou de l'image idéale du prince, dialoguent de verrière en verrière et finissent par tisser une théorie.

Le moment de l'émergence dans l'art du vitrail, à Saint-Denis et à Chartres, du thème de l'Arbre de Jessé est aussi celui qui coïncide avec un point d'équilibre de la réflexion politique, telle qu'elle sera exprimée par celui qui, à Chartres, lui donne aussi sa forme la plus subtile, Jean de Salisbury. C'est sur cette base romane, d'inspiration à la fois cistercienne et sugérienne, où s'équilibrent parfaitement les pouvoirs du roi et du prophète, que s'échafaude à l'époque gothique le nouveau message politique de la cathédrale chartraine. Il est dessiné par les verrières hagiographiques, et complété, en sa deuxième phase, par l'exaltation royale de la rose Nord: le précieux équilibre est progressivement rongé par le triomphe du roi, et Chartres, dans le stade terminal de sa construction, méritera l'appellation de cathédrale royale. 
47 En plein choeur de la cathédrale, l'illustration de la vie de Thomas Becket sonne comme un manifeste. Contrairement à Sens, seule construction contemporaine à avoir conservé ce même projet, le vitrail de Chartres ne souligne jamais, à part la scène du sacre épiscopal, les activités liturgiques ou pastorales du saint. A l'inverse, les activités diplomatiques connaissent une sorte d'inflation. Tous les cas de figures de cette tension diplomatique sont traités successivement dans la valse du roi et de l'évêque: le bon évêque et le bon roi, le bon évêque et le mauvais roi, le bon évêque et le pape, le bon évêque, le bon roi et le pape, le mauvais évêque et le mauvais roi occupent tour à tour la scène. On assiste là à une sorte de développement obsessionnel du discours politique, qui est au contraire presque banalisé sinon effacé par les imagiers sénonais. Le pape, absent du vitrail de Sens, est représenté deux fois à Chartres, qui se montre ainsi résolument proche de la papauté en même temps que de la royauté française. La valorisation de ce tandem, artisan de paix, en un temps où Philippe Auguste et Innocent III s'essaient à des affrontements feutrés, n'est sans doute pas neutre. Autre caractéristique du vitrail chartrain, l'insistance sur le caractère événementiel et dramatique, violences, fuites, confrontations, inspirations sataniques, et surtout mise en scène théâtrale du drame final. La responsabilité de Henri II est vivement soulignée, alors qu'à Sens on la passe sous silence. L'écart entre les deux interprétations est impressionnant, dans la mesure surtout où partout ailleurs au contraire de grandes affinités iconographiques rapprochent les deux cathédrales. Il souligne plus que tout la détermination politicienne des chanoines chartrains.

Exceptionnelles en elles-mêmes et dans leur assemblement, les illustrations des vies de Constantin, de Clovis, de Charlemagne, sont proposées aussi dans le choeur de la cathédrale, dans le lieu réservé aux apôtres ou aux martyrs : l'iconographie chartraine semble particulièrement engagée dans l'exaltation d'une certaine image royale.

De l'histoire de Constantin, écrite à travers celle de saint Silvestre, on retient à Chartres le soutien politique apporté par l'empereur au christianisme. Constructions d'églises, luttes contre la superstition et la magie, montrent le pape et l'empereur côte à côte dans le même souci d'édifier matériellement et spirituellement un empire très chrétien : le prince creuse lui-même la terre pour jeter les fondations de l'église Saint-Pierre de Rome, à l'endroit du tombeau de l'apôtre, et affiche l'alliance indéfectible des pouvoirs religieux et séculiers. Mais surtout, par-delà la légende, le vitrail de saint Silvestre est une composition savante qui fait cerner le récit narratif des sept prescriptions promulguées par Constantin chacun des jours de la semaine qui suivit son baptême. Elles témoignent de la faveur de l'empereur pour une Église à laquelle il se lie : obligation du culte chrétien, défense du blasphème, protection des chrétiens par la loi, reconnaissance de la hiérarchie ecclésiale, droit d'asile conféré aux églises, monopole de l'évêque pour la construction des églises, enfin autorisation de la dîme en faveur de l'église. Le tour de force des théologiens chartrains est d'avoir intégré ce texte anti-narratif s'il en fût, et aussi éloigné qu'il est possible du plaisir légendaire supposé être offert au bon peuple à travers les vitraux, à l'intérieur d'un récit parmi d'autres. La construction en est si codée et si intellectuelle que très vite vraisemblablement on n'y comprit rien : dès la fin du Moyen Age le maître verrier chargé de la restauration de la cinquième prescription la remplace par une phrase qui n'a aucun rapport avec la signification originale. Aussi bien n'est-ce pas au peuple que ces images sont destinées, mais au roi, au roi à qui on rappelle avec force le privilège ecclésial. Ce n'est plus une légende que l'on raconte avec naïveté, mais c'est le principe de l'équilibre constantinien des deux pouvoirs que les chartrains veulent 
réactiver par l'image. La légende peinte de saint Silvestre montre clairement que le roi n'est rien sans le pape. On y gomme les actions militaires au profit des actions religieuses, pour exposer les conditions de la construction de l'Église et de l'harmonie entre temporel et spirituel.

En choisissant d'illustrer, dans le choeur de leur cathédrale, l'histoire de Charlemagne, les maitres chartrains introduisent l'épopée au sein du sacré, se réclament de l'héritage des moines de Saint-Denis, renforcent la position de Philippe Auguste qui se déclare dépositaire de la gloire carolingienne : le choix est littéraire, il est politique. La qualité littéraire, par-delà la virtuosité du style ou de l'organisation des couleurs, est celle d'auteurs qui, à l'égal des jongleurs sur les routes des pèlerinages, remodèlent à leur manière des histoires déjà entendues pour en faire une oeuvre originale avec la liberté propre aux poètes. Le sens politique consiste d'abord à déterminer une place de choix pour un vitrail très audacieux, puisqu'il intègre, au milieu des plus grandes figures de martyrs ou d'apôtres, et symétriquement à Constantin, un empereur très récemment et difficilement canonisé. Il consiste à glorifier en lui, de façon indissociable, la figure du conquérant, de l'héritier du prestige mystique de l'Orient, celui du bâtisseur d'églises, du patron des croisades, du maître des routes de pèlerinages, mais aussi celui du pécheur pardonné, du pénitent soumis à l'intercession d'un saint ermite. Il consiste aussi à faire grandir la figure de Roland, jusque là à l'ombre de celle de l'empereur, en iconographie du moins, de manière à dessiner une aventure plus populaire encore, et à célébrer, conjointement avec l'image impériale, celle de l'innocent sacrifié, celle du martyr miroir du Christ. Grâce à l'exaltation de l'aventure tragique et mystique, de Roland, l'écriture de la figure royale s'équilibre, non plus par une figure prophétique, comme toutes les autres verrières le répètent, mais par celle du chevalier sans reproche.

Les « Miroirs » des princes, cette inlassable littérature qui veut dessiner le portrait idéal du roi, trouvent dans l'écriture lumineuse du vitrail une consécration sans pareille. Le discours sur la royauté y revêt un développement rare par son ampleur et son originalité. Très différent de celui que l'on pouvait trouver à Reims, il s'écrit à partir de l'image fondatrice, idéale, mystique et liturgique de l'Arbre de Jessé, de son parfait équilibre de la succession généalogique des rois et de son encadrement par une haie prophétique; il s'écrit en une fresque historique où ressortent quelques figures de proue, modèles ou repoussoirs; il s'écrit comme un pamphlet qui vilipende les tyrans et publie pour des siècles le visage honni de ceux qui ont fait les martyrs, ou comme un chant à l'honneur de la royauté française, qui sort vainqueur de ce vaste répertoire : elle hérite de Constantin, dans les images magnifiées de Clovis et de Charlemagne, et suscite la figure épique, christique, de Roland, célébré avec un honneur sans précédent dans l'espace sacré; il s'écrit en poème avec ses jeux d'oppositions, son travail sur l'implicite et la grammaire savante de ses couleurs. C'est une apologie passionnée de la suprématie de l'Église : elle fait les rois, les baptise, les sacre, les conseille, les critique, les soutient, leur pardonne. C'est aussi indistinctement une glorification de la royauté qui, à la condition de sa soumission à l'Église, trouve une célébration exceptionnelle et exemplaire à travers ces vitraux : les alliances que vivent Constantin et Silvestre, Clovis et Remi, Charlemagne et Roland, Louis VII et Becket, sont les phares qui éclairent le choeur de la cathédrale. 


\section{AUTEUR}

\section{COLETTE DEREMBLE-MANHES}

Colette DEREMBLE-MANHES est chargée de recherche au CNRS. Ce texte est un résumé de la thèse qu'elle a soutenue le 9 janvier 1984, sous la direction de Jean-Claude Schmitt. 